\title{
Resistenze europee di ieri e di oggi. ERA 2014
}

Il resoconto di ERA - European Resistance Assembly il raduno europeo della Resistenza andato in scena dall'8 all'11 maggio 2014 a Correggio, organizzato da Istoreco Reggio Emilia e Anpi Correggio. Ha visto la partecipazione di ospiti diretti della seconda guerra mondiale e la presenza di centinaia di persone arrivate da buona parte d'Europa.

The report about 2014's ERA - European Resistance Assembly is a gathering about European Resistance promoted by Istoreco and Anpi Correggio, two italian istitutes involved in History and Memory. The 2014 edition was hosted by Correggio, a little Italian town. Have attended witnesses of the II War World.

\section{L'assemblea europea della Resistenza}

Testimonianze dirette della guerra e riflessioni sul passato, ma anche su come tramandare la memoria, e su come narrare oggi la Resistenza, tutte le Resistenze. Le hanno potuto ascoltare, partecipando spesso al dibattito, le centinaia di persone arrivate da mezza Europa che dall' 8 all'11 maggio scorso si sono ritrovate a Correggio di Reggio Emilia, cittadina della Bassa che ha ospitato la terza edizione di ERA - European Resistance Assembly, il raduno europeo della Resistenza.

Un evento promosso da Istoreco Reggio Emilia e da Anpi Correggio con la collaborazione di tante realtà modenesi e reggiane, istituzionali e culturali: Comune di Correggio, Anpi provinciale Reggio Emilia, Materiale Resistente, Fondazione Fossoli di Carpi (Modena), Istituto storico di Modena, Arci Reggio Emilia, No- 
vares, Associazione giovani in Europa, Rete Spartaco, Cgil Correggio e Rumoreweb.

Quella 2014 è la terza edizione per ERA, che già nel 2013 aveva richiamato partecipanti dall'Italia, dalla Germania, dalla Francia e dalla Svizzera, oltre a testimoni diretti della Seconda guerra mondiale e nomi importanti della cultura, da Wu Ming a Massimo Zamboni e Lidia Menapace.

Si è svolta ad inizio maggio per una scelta precisa, per ricordare il sessantanovesimo anniversario della fine della guerra sul fronte europeo, arrivata l'8 maggio 1945. E proprio la lettura, in italiano, tedesco e russo del comunicato della resa incondizionata tedesca ha segnato ufficialmente l'inizio della manifestazione, nella serata di giovedì 8 maggio.

\section{Perché ERA}

ERA ha un doppio valore. Il primo autonomo, come momento di incontro e documentazione sulle Resistenze continentali, di ieri come di oggi. Il secondo quale atto finale del Viaggio della Memoria che Istoreco Reggio Emilia organizza da 15 anni coinvolgendo ogni anno più di 1.000 studenti delle scuole superiori del territorio. Ogni anno, fra febbraio e marzo, i ragazzi vanno in visita nei luoghi della guerra e dello sterminio, dopo un percorso di preparazione che prevede incontri con reduci e attività formative. Nel 2014, il Viaggio ha portato più di mille persone in visita a Berlino e ai campi di concentramento di Ravensbrück e Sachsenhausen. Per scelte precisa, l'esperienza del Viaggio non si conclude con

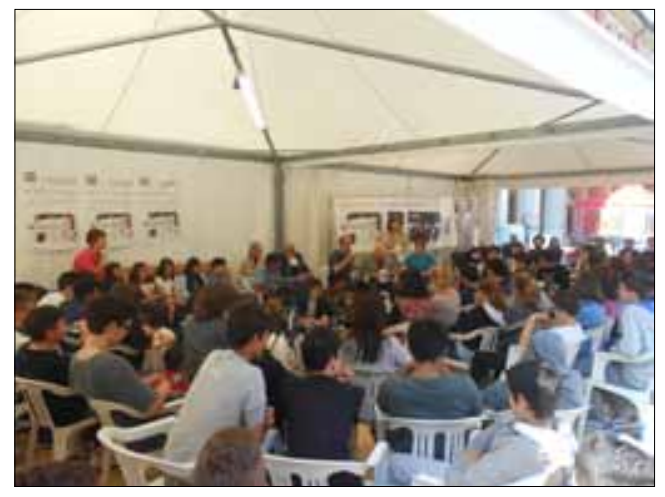

il momento all'estero, ma prosegue con un cammino di rielaborazione e condivisione che dal 2012 ha la sua conclusione collettiva proprio ad ERA, a Correggio.

Oltre agli studenti reggiani, questa edizione ha visto la partecipazione attiva anche dei loro colleghi modenesi, protagonisti nel secondo giorno della manifestazione di un colloquio con Ezio Bompani, una delle figure storiche della Resistenza locale. E uno fra i testimoni presenti al raduno. Con lui, il bielorusso-tedesco di origine ebrea Felix Lipski e il tedesco Herbert Herz, resistente ebreo in Francia, che ha parlato da Parigi grazie ad un collegamento digitale con Correggio. 
Sono i tre nomi di prestigio che si uniscono a quelli intervenuti nelle due precedenti annate di ERA. Un elenco che racconta del respiro europeo della manifestazione. Il 2012 aveva accolto il resistente tedesco Lorenz Knorr, l'ebrea francese Frida Wattenberg - che lavorò clandestinamente per tutta la guerra - i partigiani reggiani Giacomo Notari e Giacomina Castagnetti, l'ebrea lituana Fania Brancovskaya - sopravvissuta alla liquidazione del ghetto di Vilnius e poi combattente - ed Esther Bejarano, cantante ebrea tedesca uscita viva da Auschwitz e tutt'ora attiva, a 90 anni, come artista. Nel 2013 erano arrivate invece l'italiana Lidia Menapace, conosciutissima per la sua attività militante, la staffetta olandese ebrea Mirjam Ohringer ed Hans Coppi, figlio di due antinazisti tedeschi uccisi dalla Gestapo a Berlino: la madre di Hans venne giustiziata dopo aver partorito il figlio, di cui era incinta al momento della cattura.

Tutti gli interventi si svolgono in due lingue, tradotti sul momento. Sia che si tratti di persone italiane, e in questo caso le loro parole vengono tradotte in tedesco, lingua madre di molti dei partecipanti esteri. Sia che si tratti di stranieri, con il percorso inverso per gli spettatori italiani.

\section{I testimoni 2014}

Ad aprire le testimonianze 2014 è stato il modenese Ezio Bompani, un resistente che si è impegnato in prima persona e lungo tutto l'arco della sua vita per le sue idee antifasciste. Intervistato da ragazzi della scuola Lanfranco di Modena ha raccontato le scelte della sua vita, le difficoltà patite nel ventennio fascista e nel secondo conflitto mondiale, la partecipazione alla lotta di Liberazione e l'impegno politico del dopoguerra nella segreteria del Fronte della Gioventù, all'epoca diretto da Enrico Berlinguer. La mattinata si è conclusa con un momento non programmato, ma di grande emozione. Terminata la parte ufficiale, l'ultranovantenne partigiano correggese Avio Pinotti, un vero simbolo della Resistenza nella Bassa, ha raggiunto Bompiani per un breve saluto e davanti agli applausi collettivi ha consegnato al «collega» modenese una copia della sua biografia.

Venerdì 9 maggio è stato possibile ascoltare anche le parole del tedesco di origine ebraica Herbert Herz. Herz (noto come Georges-Hubert Charnay), classe 1924: un ex combattente della Resistenza francese nella FTP-MOI, membro del battaglione Carmagnola e Liberté che ha operato nella regione di Lione durante la Seconda guerra mondiale. Una storia complessa, tipica di quegli anni, la sua. La famiglia di Herz, di origine ebraica, è emigrata in Francia nel 1934 per sfuggire alle persecuzioni naziste avviate in quegli anni. Con lo scoppio della guerra, poi, la situazione è rapidamente precipitata anche in terra francese e Herz, con il suo 
nome francese, ha deciso di combattere in prima persona, a meno di vent'anni, unendosi ai partigiani lionesi, con cui ha diviso anni di missioni clandestine nella Francia prima governata da Vichy e poi occupata direttamente dalla Wehrmacht.

\section{Felix Lipski}

A chiudere il ciclo di testimonianze, nella mattinata di domenica 11 maggio, Felix Lipski, che ha voluto presenziare a tutta l'assemblea assieme alla moglie, ascoltando i racconti, le riflessioni e i progetti presentati nella quattro giorni.

Lipski è sia testimone diretto, per quanto parziale, sia di seconda generazione. Oggi cittadino tedesco, è nato l'11 maggio 1938 a Minsk, oggi Bielorussia, da una famiglia ebrea. A 3 anni compiuti da poco, il 28 giugno 1941 vive l'occupazione di Minsk da parte delle truppe tedesche e assiste alla costruzione dell'enorme ghetto dove finiscono a vivere quasi 80.000 persone. Dall'agosto 1941 all'ottobre 1943 migliaia di queste vengono torturate e uccise nel corso delle «azioni» della polizia nazista contro la popolazione ebraica, sino alla liquidazione definitiva del ghetto avvenuta nell'autunno 1943 (䀧 http://youtu.be/HWxrykX0Cz4).

Sin dal 1941 la madre di Felix, Rosa Lipskaja, partecipa all'organizzazione del gruppo clandestino di Resistenza. Nell'agosto 1943, pochi mesi prima della «liquidazione finale», fugge assieme al suo compagno Aron Fitersohn e al figlio. La coppia si unisce alla formazione partigiana di Schalom Sorin, che oltre ad un gruppo armato organizza anche un accampamento clandestino per famiglie, dove Felix trascorse gli ultimi anni della guerra.

I suoi racconti sono ovviamente figli delle testimonianze della madre, attrice protagonista seppur clandestina del conflitto, prima nel ghetto e poi nei boschi e nelle pianure bielorusse. Ma portano con sé anche un ricordo diretto, reso ancora più efficace, per quanto spesso terribilmente drammatico, dal filtro rappresentato dalla sua giovanissima età. È la storia di un bimbo di quattro anni che deve passare interi giorni chiuso in un rifugio sotterraneo, senza cibo né acqua. E che assiste alle orribili umiliazioni vissute dai genitori, dai sacrifici e da privazioni che, ascoltate dalla sua viva voce, hanno commosso ed emozionato tutti i presenti. Dalla caccia senza sosta a qualcosa da mangiare e bere, spesso risolta con metodi oltre l'emergenza, al vedere picchiati e uccisi i propri vicini di casa. Sempre con la consapevolezza che ogni momento poteva essere quello della morte, o della separazione dai propri cari.

Una situazione che anche un bimbo come Felix non poteva fare altro che percepire chiaramente, nonostante tutti gli sforzi dei genitori e degli adulti del ghetto per far vivere ai bambini una parvenza di "normalità" che evitasse loro di rimanere 
segnati ancora di più. Lo stesso Lipski è catturato, rinchiuso in un furgone blindato che l'avrebbe portato alla «liquidazione» assieme ad altre persone scelte a caso durante una delle quotidiane «pesche» dei nazisti nel quartiere ebraico. Deve la vita al coraggio di un adulto catturato con lui, che ha sfondato a calci la porta del mezzo riuscendo a far scappare tutti, approfittando di un momento di distrazione dei soldati tedeschi ma rischiando comunque tutto: la fuga è stata subito scoperta, e il gruppo di prigionieri è scappato nelle stradine del ghetto inseguito da raffiche di pallottole, senza mai sapere cosa era stato dei compagni di quella tremenda mattinata (㽎 http://youtu.be/XTZEzTlWTuU).

Queste testimonianze sono ancora più preziose e cruciali perché - l'orologio biologico su questo non mostra pietà - saranno fra le ultime disponibili "dal vivo". Una riflessione triste ma inevitabile, per chi si occupa di memoria e deve confrontarsi con il calo fisiologico delle fonti dirette. Per questo proseguono gli sforzi per mantenere traccia - tramite video, audio, resoconti - dei racconti, legati ai ragionamenti su come tenere vive queste storie così importanti. Le strade sono tante, dal coinvolgimento dei testimoni di seconda generazioni alla costruzione di archivi digitali, al maggior ruolo da dare ai luoghi. Unite poi ai ringraziamenti per chi, molto avanti con gli anni, continua a girare l'Europa e a incontrare migliaia di persone, animato da una grandissima volontà.

\section{Gli altri ospiti}

Il percorso di ERA cerca di allargare il suo raggio di azione per una volontà ben chiara e per "contrastare" questa situazione, offrendo approfondimenti sull'oggi, sulle Resistenze contemporanee, e sui modi con cui raccontare e tramandare.

Ogni giorno la manifestazione ha

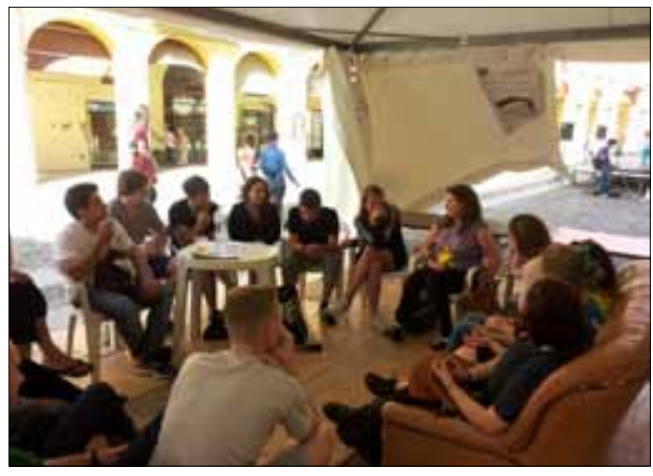
ospitato momenti di confronto fra le diverse esperienze europee partecipanti, dai militanti antifascisti agli storici, per chiudersi domenica con un dialogo fra gli studenti antifascisti tedeschi, svizzeri e italiani.

Fra le strade intraprese, vi sono poi lo sport e l'arte. Nel pomeriggio di venerdì 9 maggio una folta delegazione di ERA, ben riconoscibile dalle magliette create ad hoc per la manifestazione, hanno partecipato alla «camminata resistente» organizzata a Carpi partendo dal campo di Fossoli, uno dei principali luoghi di 


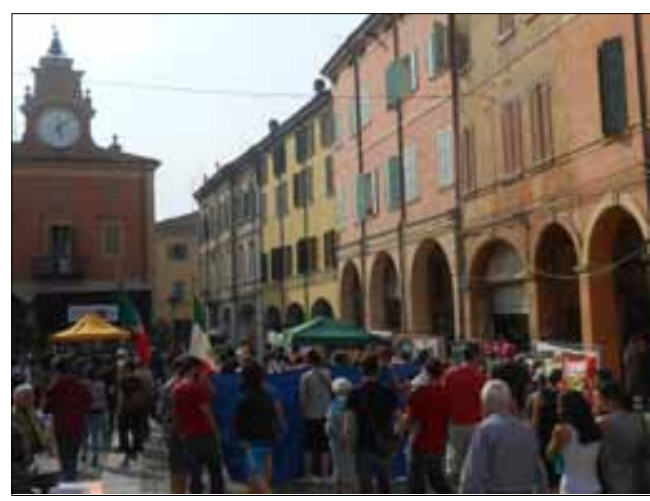

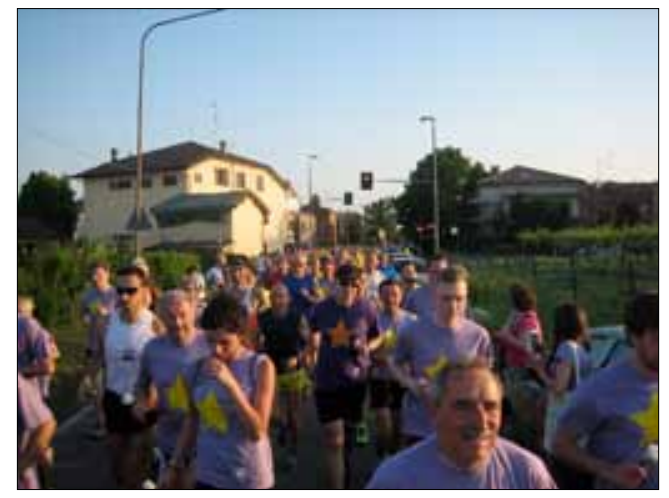

concentramento e smistamento dei prigionieri nazisti durante la guerra. Da Fossoli sono partite decine di migliaia di persone verso la Germania e verso Auschwitz, molte delle quali non sono mai tornate a casa. I marciatori di ERA hanno omaggiato il loro ricordo depositando dei fiori bianchi nelle mura esterne del memoriale oggi costruito nell'ex campo.

Largo spazio hanno avuto anche la letteratura, la musica e il teatro. Due dei momenti più seguiti sono stati gli incontri con lo scrittore Paolo Nori e con il collettivo letterario Wu Ming. Nori, venerdì pomeriggio, è partito dai testi da lui scritti dopo una visita ad Auschwitz/ Birkenau durante un Viaggio della Memoria. Due dei componenti di Wu Ming, invece, hanno parlato sabato pomeriggio davanti a parecchi spettatori, ragionando sui modi di raccontare la Resistenza. Un tema ben presente nell'opera di Wu Ming, dai primi romanzi come Q [Luther Blissett 1999] - all'epoca uscito a firma Luther Blissett - e Asce di guerra [Ravagli e Wu Ming 2005], che torna con forza anche nell'ultimo libro, L'Armata dei Sonnambuli [Wu Ming 2014], ambientato durante la Rivoluzione francese. La musica, poi, oltre a segnare le serate del raduno con vari concerti, ha offerto una suggestiva cornice ad una camminata nel centro storico di Correggio sabato pomeriggio. La Banda di Quartiere, una «marchin' band» che unisce suoni della tradizione emiliana a quelli tzigani e jazzistici, ha accompagnato questa marcia fra i vicoli, le piazze e le stradine di Correggio, inframmezzata da letture che ricordavano fatti - grandi e piccoli, spesso sconosciuti a gran parte degli abitanti della cittadina - avvenuti durante gli anni della Resistenza e dell'occupazione dentro alle mura correggesi.

Il gran finale è stato invece affidato a una storia dove l'Italia si mischia all'Africa, alle missioni imperialiste di inizio Novecento. Al centro l'attrice teatrale italoetiope Gabriella Ghermandi, che ha messo in scena una rappresentazione di Regina di fiori e di perle [Grimaldi 2011], il suo primo romanzo. Un libro dove la 
Ghermandi racconta la storia della sua famiglia, dai giorni dell' occupazione italiana del paese africano, dove il nonno italiano s'innamorò di una ragazza etiope. Un cammino fra i sogni di grandezza mussoliniani, il razzismo, i pregiudizi, i distacchi, la guerra, sino ad arrivare all'oggi, con una famiglia che galleggia in equilibrio fra due mondi

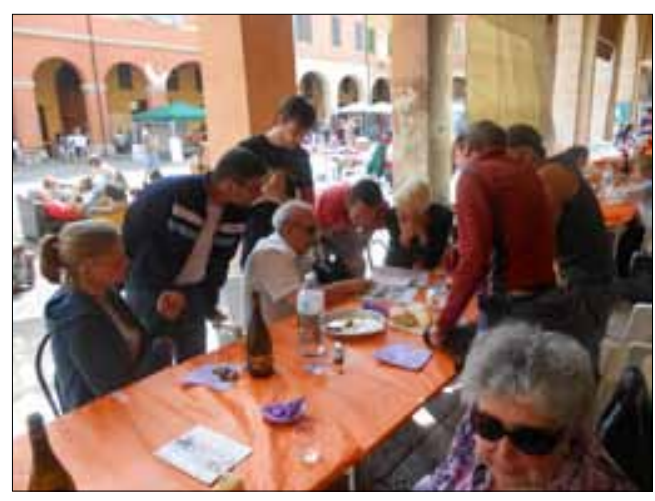
lontanissimi per tradizioni e culture.

Come in ogni raduno, non sono poi mancati i momenti dedicati al cibo e al divertimento, con apice domenica nel pranzo sotto i portici correggesi.

Si è pure pensato all'ambiente, in una modalità insolita. Gli organizzatori hanno infatti chiesto a tutti i partecipanti di adottare una piantina per azzerare il consumo di anidride carbonica emessa durante la manifestazione. Tramite un'agenzia specializzata è stata calcolata l'anidride carbonica che sarebbe stata prodotta durante la "quattro giorni», fra consumi energetici, trasporti, ristorazione, rifiuti, promozione, pernottamenti. Di seguito, sono state comprate piantine di alberi sufficienti, con loro ciclo vegetativo, a neutralizzare queste emissioni. Piantine messe a disposizione di tutti, assieme alle istruzioni per metterla a dimora nel migliore dei modi.

\section{Bibliografia}

Ghermandi G. 2011, Regina di fiori e di perle, Roma: Donzelli

Luther Blissett 1999, $Q$, Torino: Einaudi

Ravagli V. e Wu Ming 2005, Asce di guerra, Torino: Einaudi

Wu Ming 2014, L'Armata dei Sonnambuli, Torino: Einaudi

\section{Risorse on line}

Sito European Resistance Assembly http://resistance-assembly.org

FB European Resistance Assembly

https://www.facebook.com/EuropeanResistanceAssembly?fref=ts 
Twitter European Resistance Assembly https://twitter.com/ERACorreggio

Testimonianza integrale Felix Lipski, Correggio 11 maggio 2014 http://youtu.be/NUxPGhYa4Ew 